\title{
Simulação de escoamento superficial em bacias representativas na Amazônia (*)
}

\author{
Ari de Oliveira Marques Filho $\left(^{(2}\right)$, Marla de Nazaré Góes Ribeiro ${ }^{(2}$, Jesus Marden dos Santos $\left({ }^{2}\right)$ e \\ Eneas Salati ( $\left.{ }^{3}\right)$
}

\begin{abstract}
Resumo
Modelos conceituais idealizados para simular as transformações de precipitações em escoamentos, apoiados na constante evolução de técnicas numéricas e processos de otimização, provêem condições favoráveis à identificação dos principais fatores que regem essas transformações numa bacia hidrográfica e suas interrelações. Para descrever o Hidrograma Unitário de bacias hidrográficas, adotou-se um modelo constituído por uma cascata de reservatórios em série, todos com o mesmo tempo de armazenamento. A otimização dos parâmetros que caracterizam esse modelo foi efetuada através do Método de Newton-Raphson. As informações hidrológicas utilizadas foram coletadas na Bacia Representativa do rio Tarumã-Açu, Km 60 da Rodovia Manaus-Caracaraí, no periodo de setembro de 1979 a setembro de 1980
\end{abstract}

\section{INTRODUÇ̃̃o}

$O$ aproveitamento dos recursos naturais $d a$ Região Amazônica deve ser acompanhado por mecanismos de controle que definam os limites de exploração permissíveis sem prejuízo para a estabilidade do meio ambiente. No âmbito de uma bacia hidrográfica, a cobertura ve. getal tem um papel preponderante como reguladora do regime de escoamentos nos rios.

A alteração desse regime, em decorrência de explorações efetuadas na floresta, pode ser quantificada desde que sejam conhecidos modelos adequados para simulação das trans. formações de precipitações em descargas.

Um dos objetivos deste estudo é investigar a transformação da precipitação efetiva em eccoamento rápido ou superficial através de modelo conceitual, em bacias representativas na Amazônia .
Para analisar os eventos individuais de precipitação-descarga foi adaptado o algorítmo de otimização estabelecido por Marques Filho (1980) que está apoiado no modelo constituído por uma cascata de reservatórios em série, todos cum o mesmo tempo de armazenamento, propusto por Nash (1959 e 1960).

C. Modeio Nash e outros similares foram estudados por hao et al. (1972), Reed et al. (1979), O' Connell et al. (1970) entre outros, com differentes técnicas de otimização para a estimativa dos parâmetros e modificações para a simulação em bacias hidrográficas que apresentam tempos de retardo variáveis.

A determinação de valores médios que podem ser utilizados para estimativas aos escoamentos nas bacias estudadas é outro objetivo deste trabalho.

\section{Material E MÉtodos}

As informações hidrológicas foram coleta das na Bacia Representativa do rio Tarumã-Açu, pertencente ao Instituto Nacional de Pesquisas da Amazônia e iocalizada no km 60 da Rodovia Manaus-Caracaraí, correspondendo ao período de setembro de 1979 a setembro de 1980.

A distribuição dos postos pluviográficos e linigráficos na bacia é mostrada na figura 1 ; existem dois postos com pluviógrafos e linígrafos siturados ao longo do curso d'água principal e um posto pluviográfico situado na estação climatológica da bacia.

Foram utilizadas curvas chaves preliminares dos dois postos fluviográficos. As descar-

(1) - Trabalho inteiramente subvencionado pelo Conselho Nacional de Desenvolvimento Científico e Tecnológico (CNPq)

(2) - Instituto Nacional de Pesquisas da Amazônia, Manaus, AM

(3) - Centro de Energia Nuclear na Agricultura (CENA). 


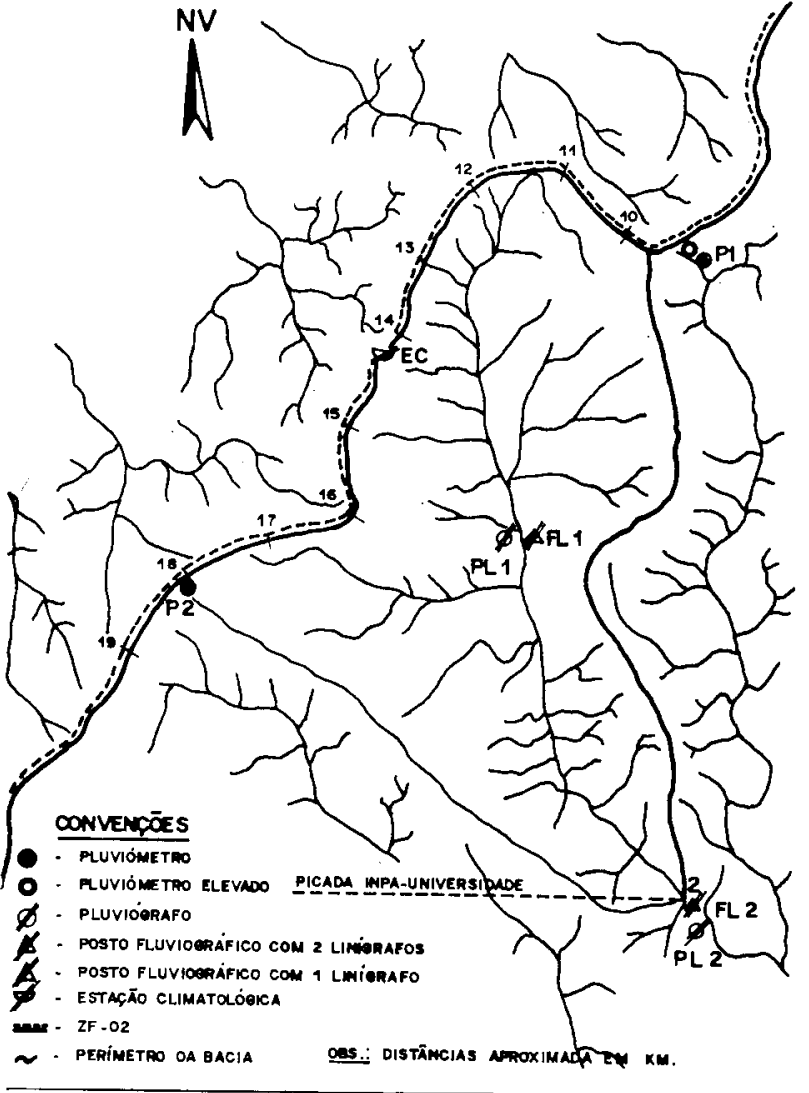

Fig. 1 - Distribuição dos postos hidrométricos na Bacia do rio Tarumã-Açu.

gas são apresentadas na forma discreta com valores definidos a intervalos de 1 hora. Em decorrência disso, as ordenadas de precipitações foram determinadas como totais precipitados nesse intervalo.

C Hidrograma Unitário (HU) proposto por Sherman em 1932 pode ser definido como "um hidrograma de escoamento superficial resultante de uma precipitação efetiva de altura unitária, distribuída uniformemente sobre a área da bacia e com uma taxa constante durante toda a sua duração".

Nesse método considera-se o escoamento total constituído por dois componentes: escoamento superficial e escoamento subterrâneo

As proposições básicas, válidas para 0 processo de transformação da precipitação efetiva em escoamento superficial, numa bacia hidrográfica, podem ser assim enunciadas:

a) A duração do escoamento superficial é sensivelmente constante para todas as precipitações de intensidades constantes e durações próximas. b) Duas precipitações com durações semeIhantes e intensidades uniformes produzem volumes diferentes de escoamento em que as taxas em tempos correspondentes apresentam a mesma proporção existente entre os volumes totais de escoamento superficial, resultantes das duas precipitações.

c) A distribuição no tempo do escoamento superficial resultante de determinado periodo de chuva é independente das precipitações anteriores porventura ocorridas.

Quando a duração da chuva efetiva se torna cada vez menor chega-se, no limite, ao conceito do Hidrograma Unitário Instantâneo (HUI), que é a resposta resultante de uma entrada instántânea. O hidrograma de escoamento supeificial numa bacia hidrográfica, correspondente a um dado período de precipitação, pode ser determinado pela superposição dos HUI resultantes de cada ordenada de precipitação efetiva $x(\tau)$. Essa soma dos HUI, que está ilustrada na figura 2, é representada pela integral de convolução.

$$
y(t)=\int_{j_{0}}^{t \leq t_{0}} x(\tau) h_{0}(t-\tau) d \tau
$$

onde,

$$
\begin{aligned}
\mathrm{y}(\mathrm{t}) & \rightarrow \text { descarga ocorrida no instante } t . \\
\mathrm{t}_{0} & \rightarrow \text { duração da precipitação. } \\
\mathrm{h}_{0}(\mathrm{t}-\tau) & \rightarrow \text { ordenadas do HUl. } \\
\mathrm{t} & \rightarrow \text { variável que representa o tem- } \\
& \text { po. }
\end{aligned}
$$

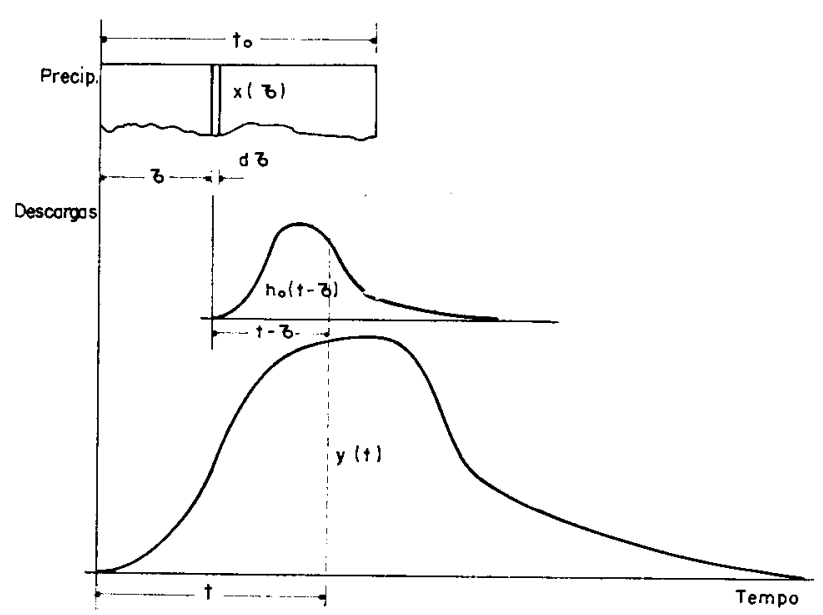

Fig. 2 - Convolução. 
Quando as ordenadas de chuva $x(\tau)$ são iguais a uma constante $(1 / T)$ e constituem um volume unitário de precipitação, a equação 1 toma a forma

$$
h_{T}(t)=\frac{1}{T} \int_{0}^{T} h_{0}(t-\tau) d \tau
$$

onde,

$$
\begin{aligned}
h_{T}(t) \rightarrow & \text { ordenadas do HU corresponden- } \\
& \text { te a precipitação com duração } \\
& \text { de } T \text { horas (HUT) }
\end{aligned}
$$

A equação 2 pode ser transformada para a forma

$$
h_{T}(t)=\frac{1}{T} \int_{t-T}^{t} h_{0}(\tau) d \tau
$$

que também pode ser utilizada para o estabelecimento do HUT.

A convolução indicada na equação 1 só pode ser efetuada quando as precipitações são conhecidas continuamente. Nos casos práticos correntes, porém, os dados de precipitações são armazenados na forma de histogramas, com totais precipitados em intervalos de 1,2, 3 horas, etc. Essa simplificação nos registros torna necessária a utilização de hidrogramas unitários correspondentes às durações das precipitações usadas nesses histogramas. Nesse caso, o hidrograma resultante é determinado pela convolução discreta entre a precipitação na forma de histograma e o HUT.

$$
y(t)=\sum_{J=1}^{J=t \leq t_{c}} x(J) \cdot h_{T}(t-J+1)
$$

onde.

$$
\begin{aligned}
& j \quad \rightarrow \text { número da ordenada do his- } \\
& x(J) \rightarrow \text { ordenada do histograma de } \\
& \text { precipitações } \\
& h_{T}(t-J+1) \rightarrow \text { ordenada do HUT }
\end{aligned}
$$

Nash (1959) sugeriu um modelo para representar o HUI, constituído por $n$ reservatórios lineares $\mathrm{\epsilon m}$ série todos com o mesmo tempo de armazenamento $K$. As ordenadas do HUI segundo esse modelo săr dadas por

$$
h_{i j}(t)=\frac{1}{K}\left(\frac{t}{K}\right) \cdot \frac{e^{-t / K}}{T n}
$$

$\mathrm{Na}$ aplicação do modelo, Nash utilizou o método da comparação dos momentos (determinou os momentos da entrada e da resposta e relacionou-os aos momentos do HUI) para a determinação dos valores ótimos dos parâ. metros $n$ e $k$ em cada evento precipitação--descarga analisado. A otimização realizada dessa maneira, além de exigir extensos cálculos, pode conduzir a resultados irreais.

No trabalho desenvolvido por Marques $\mathrm{Fi}$ Iho (1980) foi estabelecido um algorítmo que pesquisa os valores ótimos dos parâmetros minimizando a soma dos quadrados das diferenças entre as vazões observadas $\Theta$ as vazões calculadas segundo o modelo. O mínimo da função objetiva é determinado através do Método de Newton-Raphson adaptado a essa finaiidade.

O Método de Newton-Raphson foi estabelecido inicialmente para a determinação das raízes de uma equação. As adaptações para transformá-lo num método de otimização são imediatas.

$$
\begin{aligned}
& \text { A função objetiva } \\
& z=\sum_{t=1}^{M} \quad\left(y_{0}(t)-y(t){ }^{2}\right)
\end{aligned}
$$

onde.

$$
\begin{aligned}
& y_{0}(t) \rightarrow \text { descargas observadas (total de } \\
& y(t) \rightarrow \text { descargas calculadas segundo } 0
\end{aligned}
$$

apresentará um ponto crítico (máximo, mínimo ou outro ponto particular) quando as derivadas parciais de primeira ordem emi relação aos pa- 
râmetros forem nulas. A função objetiva $Z$ é dependente dos parâmetros $n$ e $K$, pois $\mathrm{y}(\mathrm{t})$ é caracterizada pelos mesmos. Então,

$$
\begin{aligned}
& S_{1}=\frac{\partial Z}{\partial n}=f_{1}(n, K)=0 \\
& S_{2}=\frac{\partial Z}{\partial K}=f_{2}(n, K)=0
\end{aligned}
$$

são as condições necessárias para que seja encontrado um ponto crítico de $Z$. Para se chegar aos valores $n$ e $K$ que anulam as derivadas, devem ser estimados valores iniciais para os parâmetros, $n^{0}$ e $K^{c}$. As diferenças entre os valores estimados e os valores procurados são expressas por

$$
\begin{aligned}
\Delta \mathrm{n}^{0} & =\mathrm{n}-\mathrm{n}^{0} \\
\Delta \mathrm{K}^{0} & =\mathrm{K}-\mathrm{K}^{0}
\end{aligned}
$$

As derivadas parciais podem ser apresentadas na forma

$$
\begin{aligned}
& S_{1}=f_{1}\left(n^{0}+\Delta n^{0}, K^{0}+\Delta K^{0}\right)=0 \\
& S_{2}=f_{2}\left(n^{0}+\Delta n^{0}, K^{0}+\Delta K^{0}\right)=0
\end{aligned}
$$

Expandindo em série de Taylor essas duas igualdades, têm-se

$$
\begin{aligned}
& S_{1}+\frac{\partial S_{1}}{\partial n} \cdot \Delta n^{0}+\frac{\partial S_{1}}{\partial K} \cdot \Delta K^{0}+\ldots=0 \\
& S_{2}+\frac{\partial S_{2}}{\partial n} \cdot \Delta n^{0}+\frac{\partial S_{2}}{\partial K} \cdot \Delta K^{0}+\ldots=0
\end{aligned}
$$

Desprezados os termos das séries superiores à primeira ordem, forma-se um sistema de equações nas incógnitas $\Delta n^{0}$ e $\Delta K^{0}$, pois os outros elementos $\left(S_{1}, S_{2}, \partial S_{1} / \partial n, \ldots\right.$ etc. $)$ são calculados numa primeira aproximação, pelos valores iniciais $\mathrm{n}^{0}$ e $\mathrm{K}^{0}$.

$\mathrm{Na}$ segunda iteração os parâmetros terão os seus valores corrigidos por

$$
\begin{aligned}
& \mathrm{n}^{1}=\mathrm{n}^{0}+\Delta \mathrm{n}^{0} \\
& \mathrm{~K}^{1}=\mathrm{K}^{0}+\Delta \mathrm{K}^{0}
\end{aligned}
$$

Com esse novo par de valores, $n^{1}$ e $K^{1}$. procede-se de forma idêntica àquela descrita para o par $n^{0}$ e $K^{0}$, resultando agora correções para $\mathrm{n}^{1} \mathrm{e} \mathrm{K}^{1}$.

Esse proceclimento é repetido até que as correções sejarr inferiores à precisão estabelecida para os valores de $n$ e $K$.

Encuntrado um ponto crítico de $Z$, deve ser verificado se o mesmo corresponde a um mínime da função $Z$. As condições que devem ser satisfeitas são
a) $\left.\mid\left(\frac{\partial^{2} Z}{\partial n \partial K}\right)^{2}-\frac{\partial^{2} Z}{\partial n^{2}} \cdot \frac{\partial^{2} Z}{\partial K^{2}}\right\}<0$
b) $\quad \frac{\partial^{2} Z}{\partial n^{2}}>0$ e $\frac{\partial^{2} Z}{\partial K^{2}}>0$

A alteração efetuada no algorítmo de otimização, estabelecido no trabalho anteriormente citado, foi o equacionamento do HUT e suas derivadas na forma discreta, em concordância às descargas observadas.

A ordem sequencial de operações no programa, estabelecido para a determinação dos parâmetros $n$ e $K$ do modelo, em cada evento precipitação-descarga, é mostrada a seguir :

1 - Determinação das descargas de escoamentos superficiais observadas.

2 - Determinação da precipitação efetiva correspondente.

3 - Estimativas dos valores iniciais para os parâmetros $n$ e $K$.

4 - Determinação do HUT e de suas derivadas.

5 - Obtenção da função objetiva e de suas derivadas.

6- Cálculo das correções parciais necessárias aos parâmetros (Método de NewtonRaphson) .

Se essas correções forem inferiores à precisão desejada, um ponto crítico de $Z$ foi encontrado. Caso contrário, os passos 4 a 6 devem ser repetidos, com os valo. res corrigidos no final de cada iteração.

7 - Verificaçãe se o ponto crítico corresponde ao mínimo procurado (análise das derivadas). 
Ocorrendo fracasso nessa verificação um novo ponto de partida deve ser escolhido apoiado na análise da conformação da função objetiva. Esta análise pode ser incluída como uma sub-rotina do programa principal.

O método adotado para a separação dos escoamentos foi aquele proposto por Linsley et al. (1958). Determinado o escoamento superficial, a precipitação efetiva é calculada através do processo conhecido como "índice $\phi^{\prime \prime}$.

\section{RESUlTADOS OBTIDOS E DISCUSSÃO}

$O$ posto fluviográfico $n^{0} 1$ define uma bacia com área estimada de $11,5 \mathrm{~km}^{2}$. Foram selecionadas nesse posto cinco cheias, nas quais a característica comum é a duração de 1 hora da precipitação efetiva. Os hidrogramas, ob. servado e calculado em cada cheia, são mostrados nas figuras $3,4,5,6$ e 7 . O models utilizado gerou vazões de pico inferiores àque-

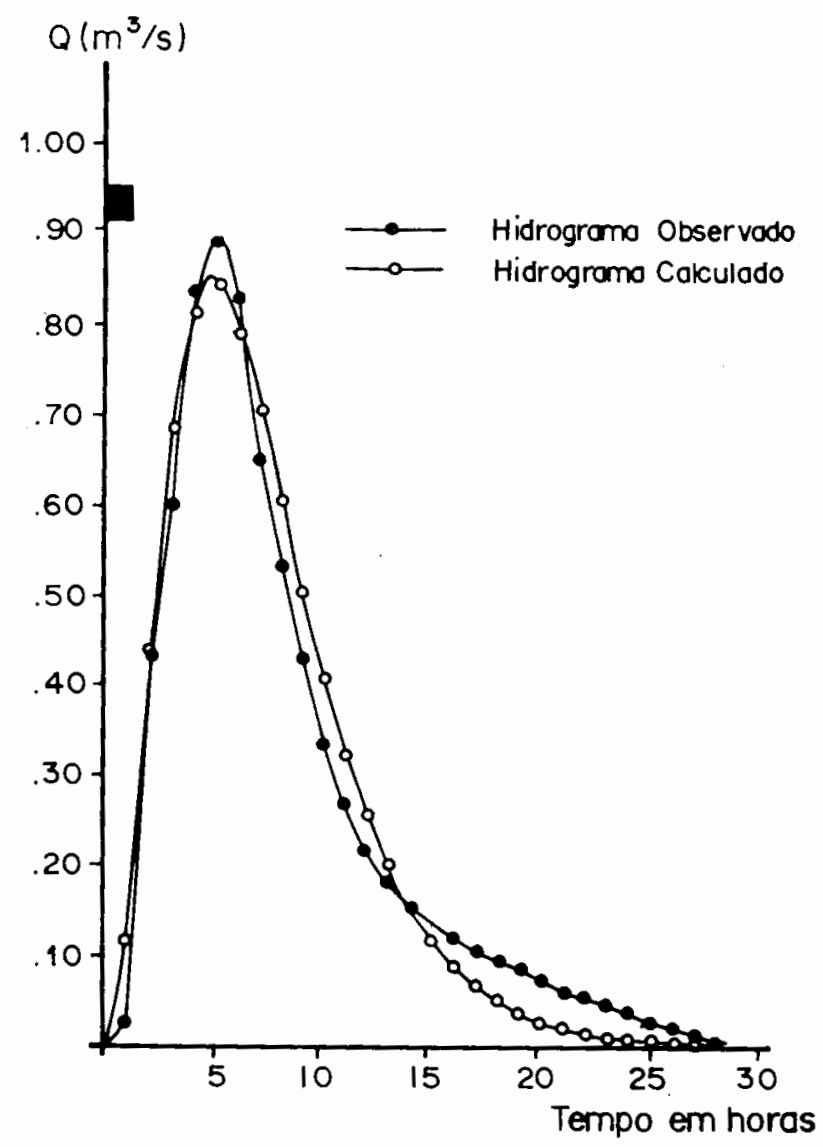

Fig. 3 - Otimizagão; cheia de 31-05-80; PF 1.

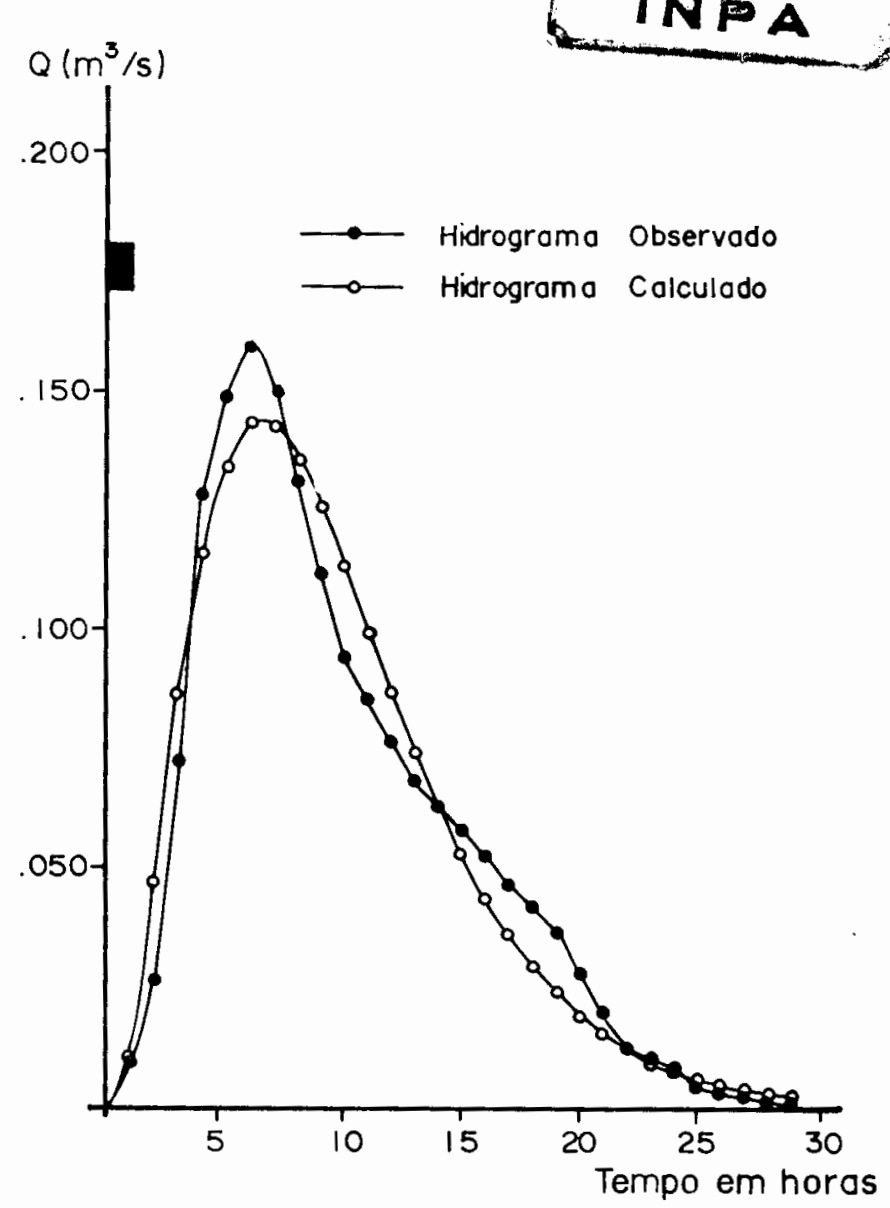

Fig. 4 - Otimização; chela de 10-05-80; PF 1.

las observadas em todos os eventos. Não se pode perder de vista, no entanto, que este fato pode estar relacionado com a função objetiva; nesta análise, essa função representa uma otimização volumétrica sem a ponderação das parcelas conforme a magnitude da descarga.

As figuras mostram simulações aceitávels, e as discrepâncias nas vazōes de pico situaram-se abaixo de $10 \%$. Os parâmetros do HUI correspondentes a cada cheia e os valores médios resultantes, válidos para essa bacia hidrográfica, aparecem na tabela 1 .

G posto fluviográfico $n^{9} 2$ registra as descargas de uma área de contrıbuição estimada em $23 \mathrm{~km}^{2}$. Os seis eventos analisados nesse posto são mostrados nas figuras $8,9,10,11$. 12 e 13.

As cheias de 20-11-79 (figura 9) e de 31-05-80 (figura 12) apresentam aspectos semelhantes nos hidrogramas observados e evi- 


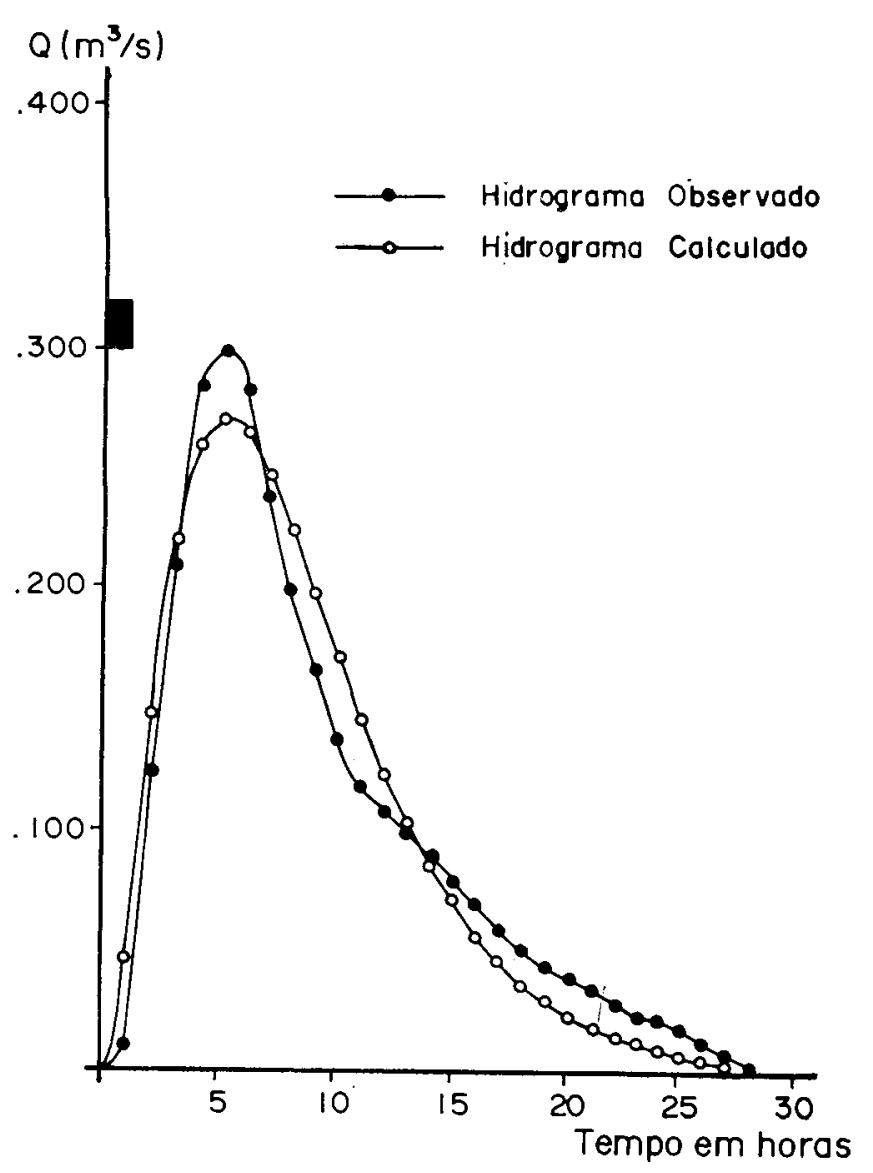

Fig. 5 - Otimização; cheia de 04-06-80; PF 1.

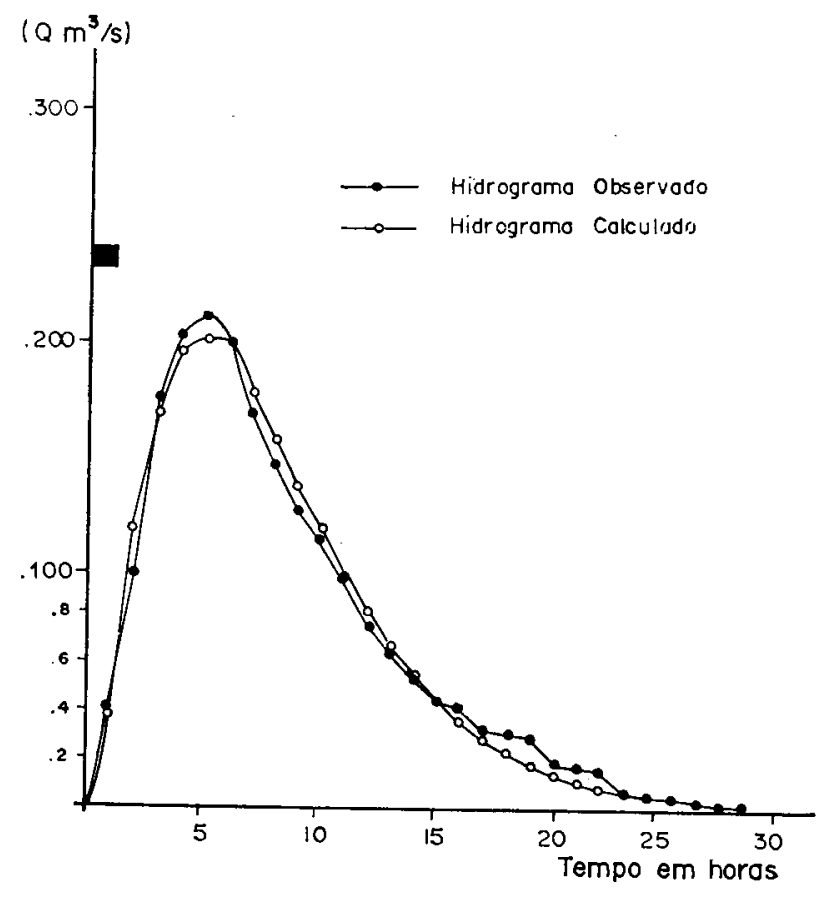

Fig. 6 - Otimização; cheia de 04-07-80; PF 1.

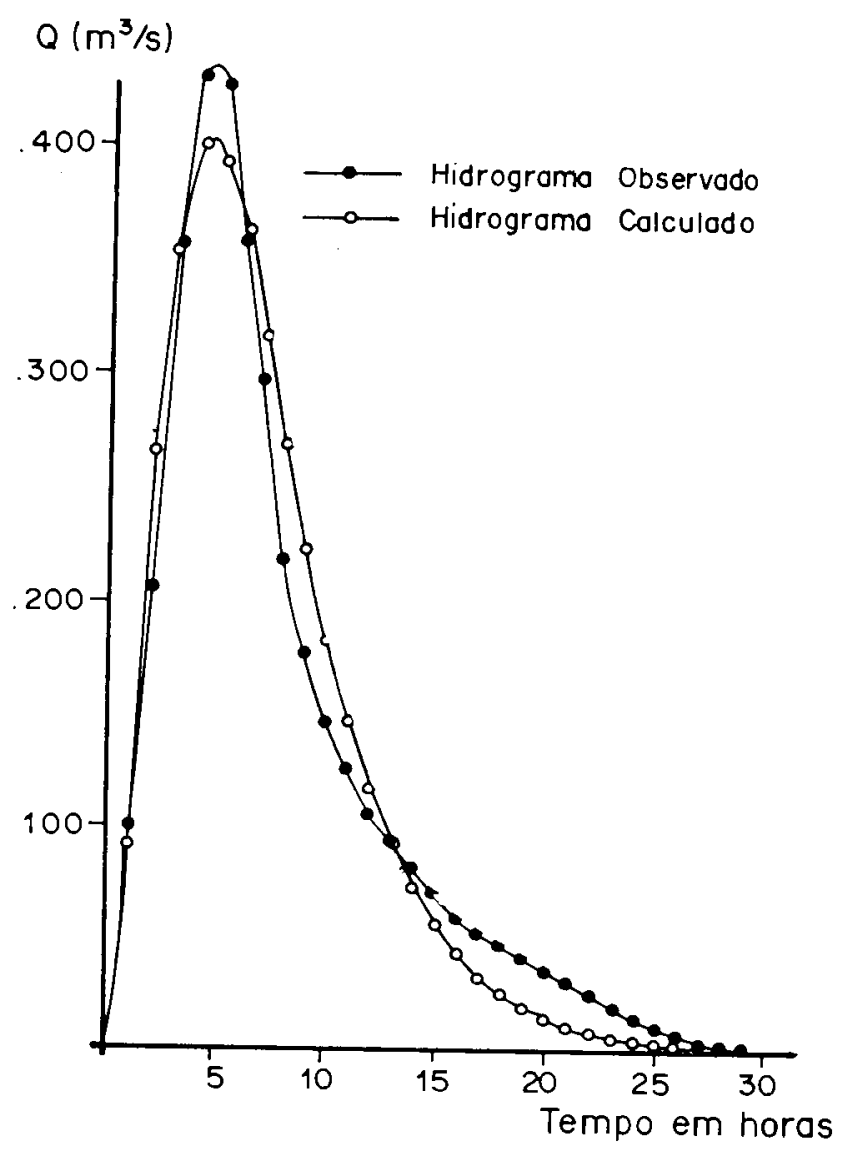

Fig. 7 - Otimização; cheia de 10-08-80; PF 1.

denciam possiveis problemas nos registros linigráficos do posto. Nesses dois eventos, os hidrogramas observados a calculados segundo o medelo mostram diferenças acentuadas nos tempos de ocorrência das vazões de pico.

TABELA 1 - Resultados obtidos no PF n.: 1.

\begin{tabular}{l|c|c|c}
\hline Data da cheia & $\begin{array}{c}n \\
\text { (adim.) }\end{array}$ & $\begin{array}{c}\mathrm{K} \\
\text { (horas) }\end{array}$ & $\begin{array}{c}\text { n.K } \\
\text { (horas) }\end{array}$ \\
\hline $10-05-80$ & 2,79 & 3,29 & 9,18 \\
$31-05-80$ & 2,65 & 2,57 & 6,81 \\
$04-06-80$ & 2,34 & 3,43 & 8,03 \\
$04-07-80$ & 2,26 & 3,41 & 7,71 \\
$10-08-80$ & 2,29 & 2,89 & 6,62 \\
\hline Parâmetros & 2,47 & 3,12 & 7,67 \\
\hline
\end{tabular}


As demais cheias analisadas produziram simulações com qualidade comparável àquela encontrada nos eventos do PF no 1. A tabela 2 agrupa os resultados alcançados na bacia de contribuição do posto fluviográfico $n^{0} 2$.

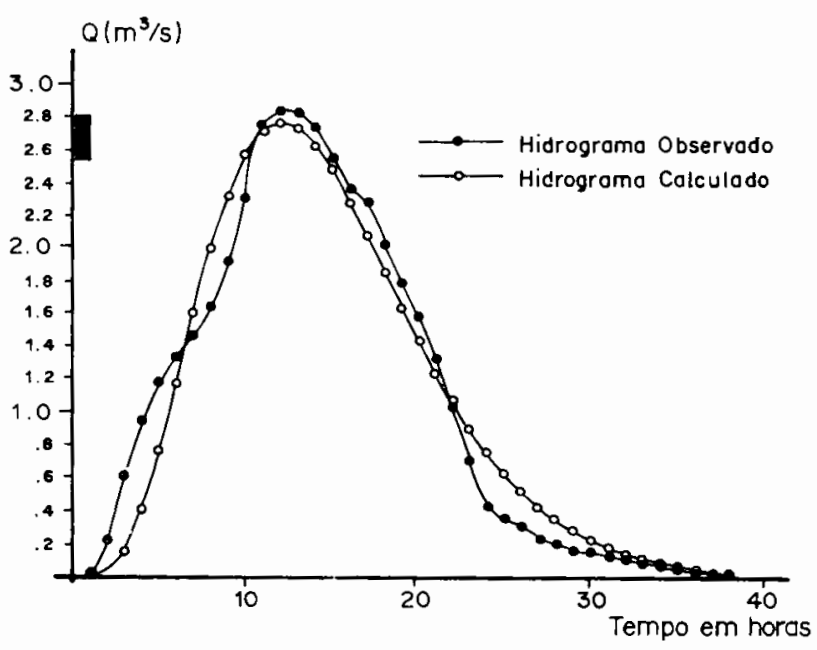

Fig. 8 - Otimização; cheia de 18-12-79; PF 2.

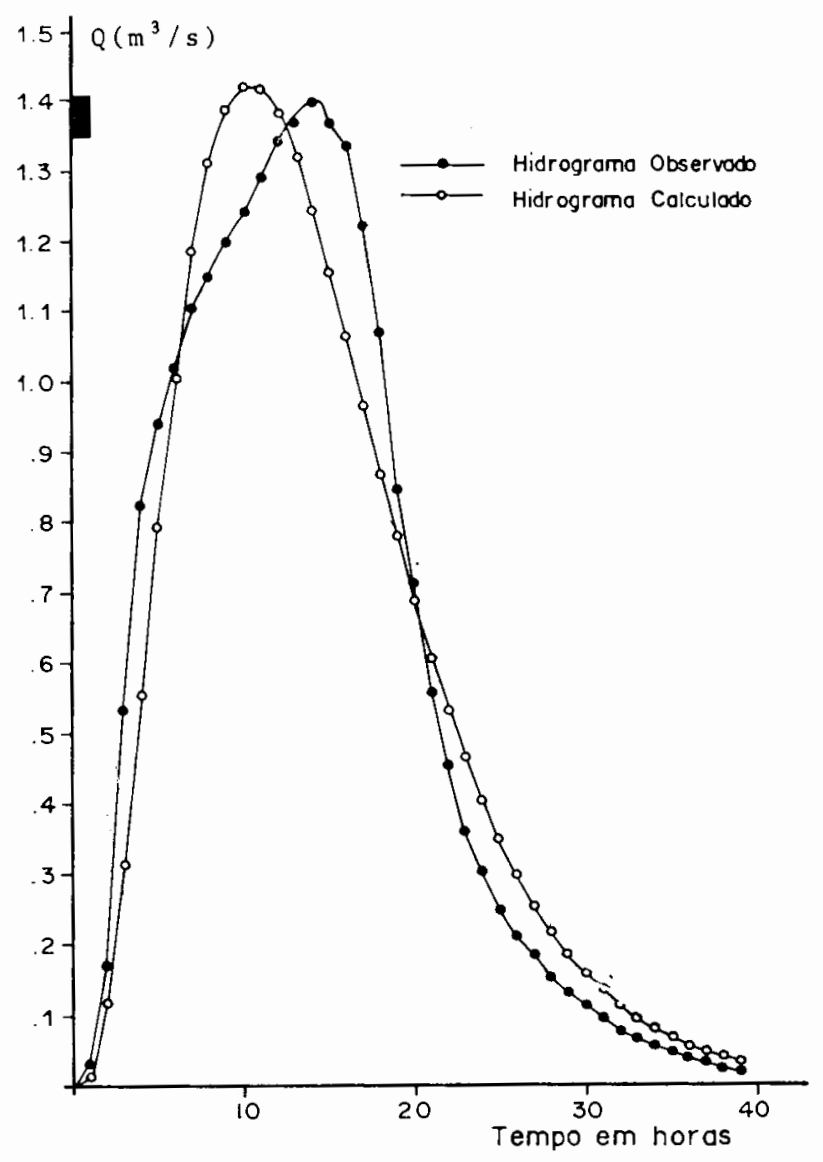

Fig. 9 - Otimização; cheia de 20-11-79; PF 2,

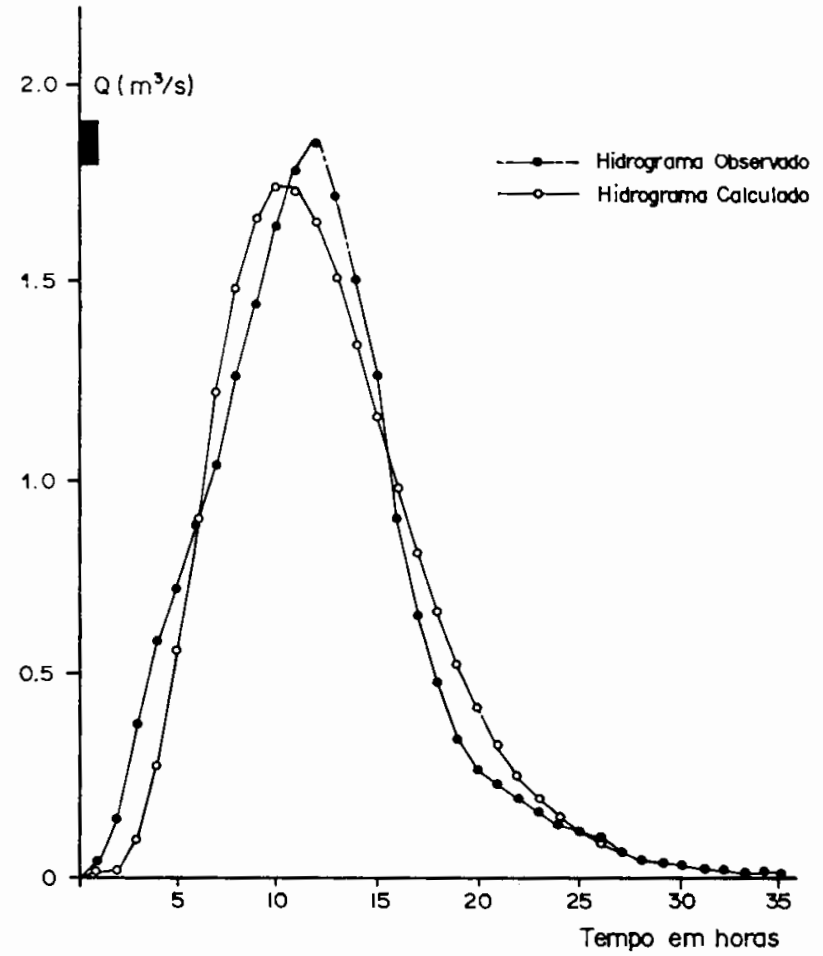

Fig. 10 - Otimização; cheia de 04-02-80; PF 2.

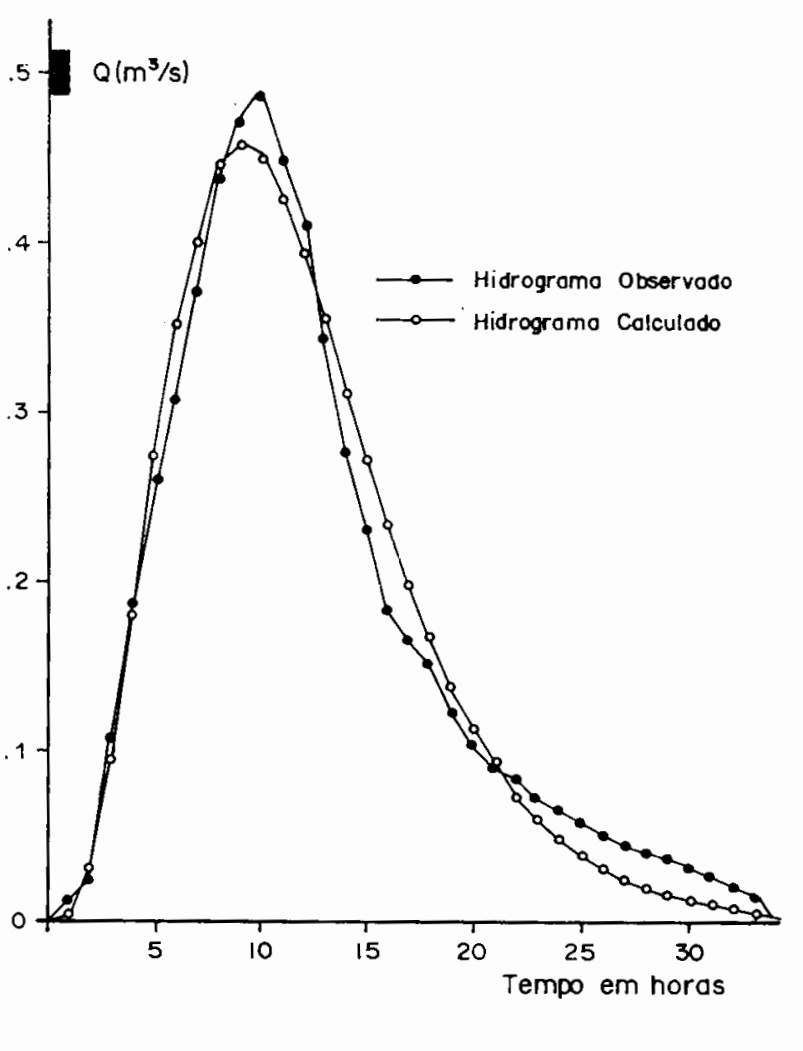

Fig. 11 - Otimização; cheia de 10-08-80; PF 2. 


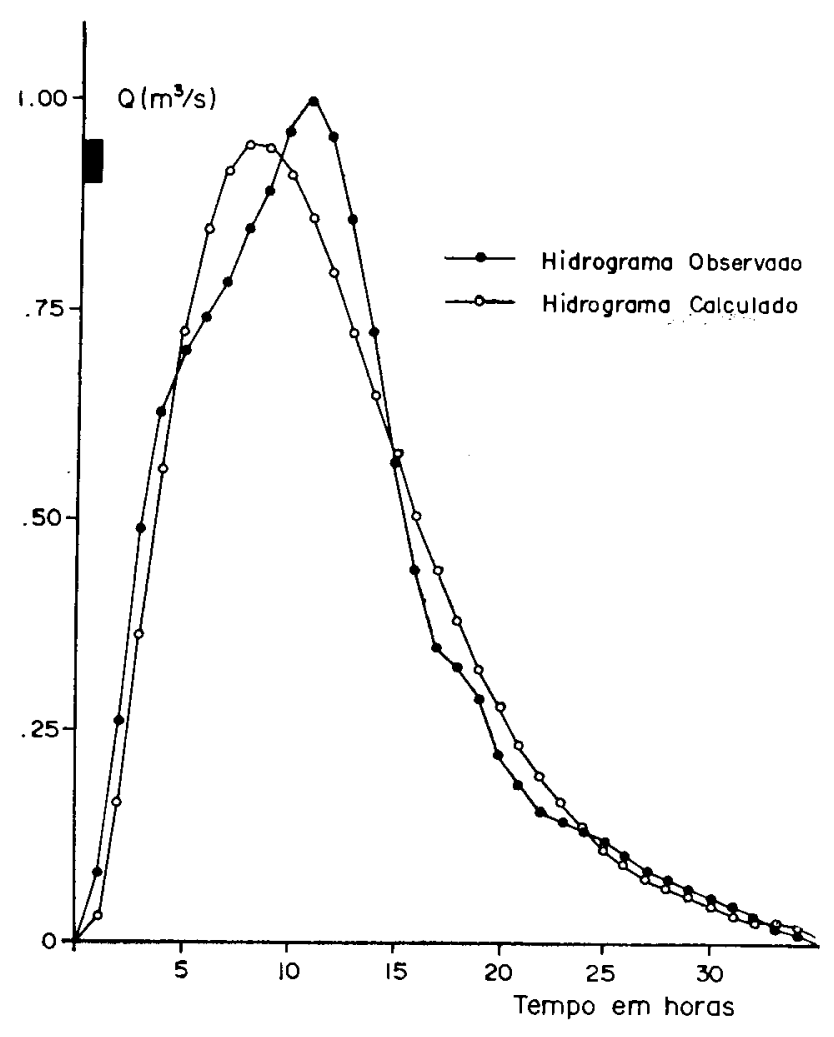

Fig. 12 - Otimização; cheia de 31-05-80; PF 2.

TABELA 2 - Resultados obtidos no PF n: 2.

\begin{tabular}{l|c|c|c}
\hline Data da cheia & $\begin{array}{c}n \\
\text { (adim.) }\end{array}$ & $\begin{array}{c}\mathrm{K} \\
\text { (horas) }\end{array}$ & $\begin{array}{c}\mathrm{n} . \mathrm{K} \\
\text { (horas) }\end{array}$ \\
\hline $20-11-79$ & 3,37 & 4,14 & 13,95 \\
$18-12-79$ & 4,93 & 2,94 & 14,49 \\
$04-02-80$ & 5,76 & 2,07 & 11,92 \\
$24-02-80$ & 5,17 & 2,15 & 11,12 \\
$31-05-80$ & 3,08 & 3,74 & 11,52 \\
$10-08-80$ & 4,04 & 2,82 & 11,39 \\
\hline Parâmetros & 4,39 & 2,98 & 12,40 \\
Médios & & & \\
\hline
\end{tabular}

\section{Conclusões}

Os valores médios dos parâmetros que caracterizam o Mcdelo Nash, foram determinados em duas sub-bacias do ric Tarumã-Açu. Esses valores identificam as transformações da precipitação efetiva em escoamento rápido para a situação de floresta inexplorada.
O tempo de retardo médio do HUI (produto n.K) alcançou 7,7 horas na bacia do PF $n^{0} 1 \mathrm{e}$ 12,4 horas na bacia do PF no 2 .

Em todos os eventos estudados a convergência para o mínimo da função objetiva não ultrapassou cinco iterações, confirmando as características favoráveis do Método de Newton-Raphson, quando utilizado como processo de otimização.

A complementação com informações sobre evapotranspiração, intercepçãc e infiltração ocorridas na bacia, permitirá a análise de chelas complexas, e determinará melhor qualidade nos valores médios dos parâmetros.

Em todas as cheias analisadas as diferenças entre as vazões de pico, observadas e calculadas, foram inferiores a $10 \%$.

Conclui-se que projetos $\epsilon$ previsões de cheias nas bacias estudadas podem se apoiar nos resultados alcançados no trabalho.

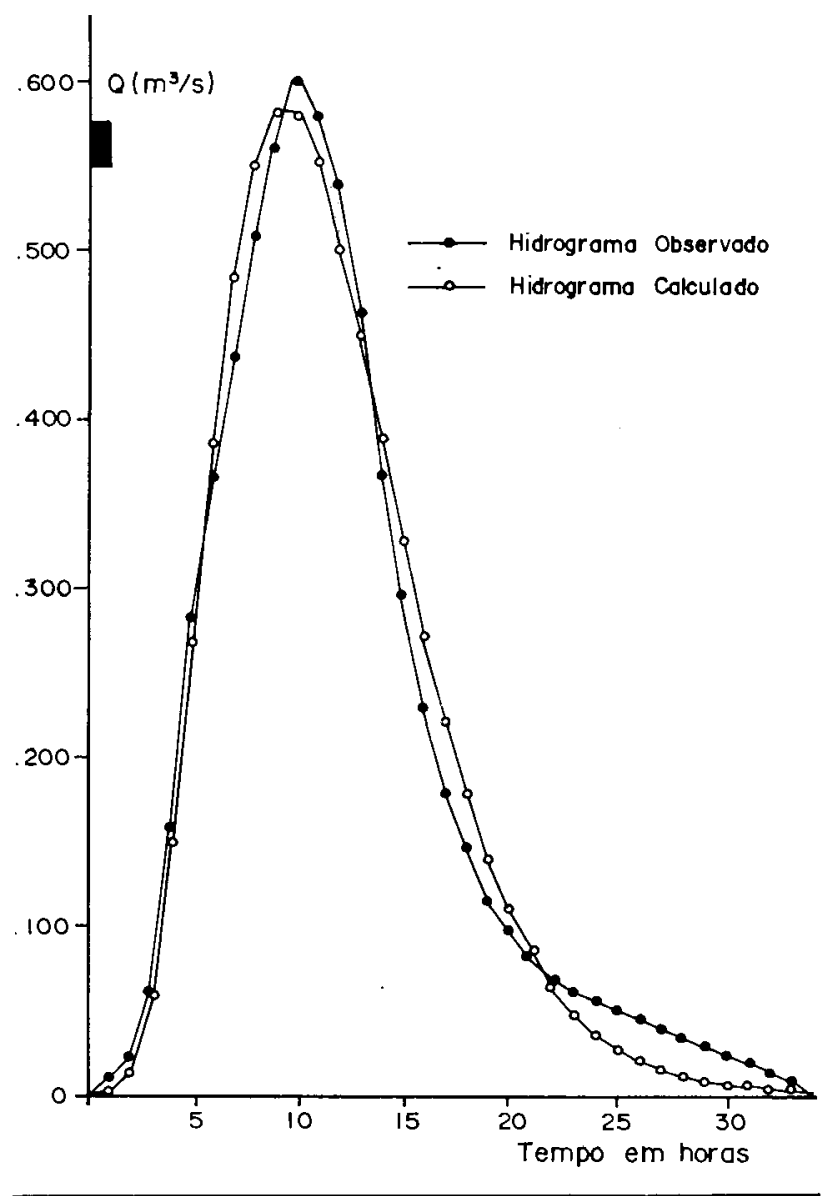

Fig. 13 - Otimização; cheia de 24-02-80; PF 2. 


\section{Agradecimentos}

Os autores agradecem às auxiliares técnicas Maria Solange da Graça Pinheiro e Valdelice Pereira da Silva pela colaboração prestada no processamento dos dados hidrológicos.

\section{SUMMARY}

Concpetual models developed to simulate runoff from rainfall, based on numerical technics and optimization processes, make it possible to identify the main factors that regulate rainfall-runoff relationship in a watershed.

It was adapted a model consisting of a cascade of equal reservoirs in serie to describe the watershed unit hydrograph. The parameters optimization that characterizes the model was done by the Newton-Raphson Method.

Al the hydrological informations was collected in the Representative Watershed of Tarumã-Açu River, Km 60 of Manaus - Caracarai Road and corresponds to the period of Sept-1979 to Sept-1980.

\section{REFERENCIAS BIBLIOGRÁFICAS}

LINSLEY, R.K.; KOHLER, M.A.; PAULHUS, Joseph L.H. 1958 - Hidrology for engineers. New York, McGraw-Hill. 340p.
MARQUES FILHO, Ari de Oliveira

1980 - Regionalização de modelo de escoamento superficial para dados não homogêneos. Porto Alegre, Instituto de Pesquisas Hidráulicas da UFRGS. 120f. Diss. (mestr. hidrol. apl.) UFRGS - Curso Pós-Grad. Hidrol. Apl., Porto Alegre, BR-RS, 1980.

NASH, J.E.

1959 - Systematic determination of unit hydrograph parameters. Journal of Geophysical Research, Washington, 64 (1): 111-5, Jan.

1960 - A unit hydrograph study, with particular reference to British catchment. Proceedings. Institution of Civil Engineers, London, 17: 249-82, Nov.

O'CONNELL, P.E.; NASH, J.E.; FARRELL, J.P.

1970 - River flow forecasting through conceptual models. Journal of Hydrology, Amsterdam, 10: $317-329$.

RAO, R.A.; DELLEUR, J.W.; SARMA, B.

1972 - Conceptual Hydrologic Models for Urbanising basin. Journal of the Hydraulic Division, New York, 99 (7): 1205-20.

REED, D.W.; JOHNSON, P.; FIRTH, J.M.

1975 - A non-linear rainfall-runoff model, providing for variable lag time. Journal of Hydrology, Amsterdam, 25: 295-305.

(Aceito para publicação em 05/04/82) 